\title{
Fuzzy-logic tuned constant modulus algorithm and soft decision-directed scheme for blind equalisation
}

\author{
S. Chen ${ }^{\text {a }}{ }^{*}$, B.L. Luk ${ }^{\text {b }}$, C.J. Harris ${ }^{\text {a }}$, L. Hanzo ${ }^{\text {a }}$ \\ a School of Electronics and Computer Science, University of Southampton, Southampton SO17 1BJ, UK \\ ${ }^{\mathrm{b}}$ Department of Manufacturing Engineering and Engineering Management, City University of Hong Kong, Hong Kong, China
}

\section{A R T I C L E I N F O}

Article history:

Available online 29 August 2009

\section{Keywords:}

Blind equalisation

Quadrature amplitude modulation

Constant modulus algorithm

Soft decision-directed adaptation

Minimum mean square error

Adaptive step size

Fuzzy logic

\begin{abstract}
A B S T R A C T
This contribution revisits blind equalisation for high-order quadrature amplitude modulation systems using a low-complexity high-performance concurrent constant modulus algorithm (CMA) and soft decision-directed (SDD) scheme. A fuzzy-logic (FL) tuning unit is designed to adjust the step size of the CMA, and the potential benefits of using this adaptive step size approach are investigated. Simulation results obtained confirm that faster convergence can be achieved with this FL assisted CMA and SDD scheme, compared with the previous constant step-size CMA and SDD scheme. Quantitatively, the former requires several thousands fewer samples to converge to the same steady-state solution achieved by the latter.
\end{abstract}

(C) 2009 Elsevier Inc. All rights reserved.

\section{Introduction}

In certain communication systems, training is infeasible and blind equalisation provides a practical means for combating the detrimental effects of channel dispersion in such systems. Since no training sequence is needed, blind equalisation in general improves system bandwidth efficiency. For systems employing high-throughput quadrature amplitude modulation (QAM) signalling [1], the constant modulus algorithm (CMA)-based equaliser [2-5] is a popular low-complexity equalisation scheme. The CMA is known to be very robust to imperfect carrier recovery. Many studies have investigated the performance and convergence behaviour of the CMA, see for example the review in [6]. A serious problem associated with the CMA is that its steady-state mean square error (MSE) may not be sufficiently low for the system to achieve an adequate symbol error rate (SER) performance. A possible solution is to switch to a decision-directed (DD) adaptation after the convergence of the CMA, which should be able to minimise the residual CMA steady-state MSE [7] and therefore achieves a performance close to the minimum MSE (MMSE) solution. However, as pointed out in [8], a successful switch to the DD adaptation requires that the CMA's steady-state MSE must be sufficiently small. In practice, such a low level of MSE may not be achievable by the CMA scheme. An alternative is to adopt a composite cost function, which consists of the CMA cost function and the decision-based MSE [9]. However, the weighting of the two component cost functions must be carefully chosen, which can be difficult to do.

The authors of [8] have suggested an interesting solution to overcome the above-mentioned problem of the CMA-based blind equalisation. Instead of switching to a DD adaptation after the CMA has converged, they have proposed to operate a DD equaliser in parallel with a CMA equaliser. The weight adaptation of the DD equaliser follows that of the CMA equaliser and, to avoid error propagation due to incorrect decisions, the DD adjustment only takes place if the CMA adaptation is deemed to have achieved a successful adjustment of the equaliser weight vector with a high probability. At a cost of

\footnotetext{
* Corresponding author. Fax: +44 (0) 2380594508.

E-mail address: sqc@ecs.soton.ac.uk (S. Chen).
} 
slightly more than doubling the complexity of the simple CMA, this combined CMA and DD equaliser is capable of achieve a dramatic improvement in equalisation performance over the CMA [8]. More recently, a novel combined CMA and soft DD (SDD) blind equaliser has been proposed [10-12], which achieves a faster convergence and has simpler implementation than the combined CMA and DD scheme of [8]. This combined CMA and SDD scheme operates a CMA-based equaliser and the last-stage SDD equaliser of [13] in a truly parallel manner. It is capable of achieving an equalisation performance that is close to the MMSE equalisation solution based on the perfect channel information and, thus offers a low-complexity high-performance technique for blind equalisation of high-order QAM channels.

For a stochastic gradient adaptive algorithm, such as the training-based least mean square (LMS), the step size must be sufficiently small to avoid divergence. Within the range of step size values that ensures convergence, a smaller step size achieves better steady-state performance at the expense of slower convergence speed, while a larger step size improves convergence speed with poorer steady-state performance [14]. A constant step-size LMS algorithm thus has to trade off between the steady-state performance and convergence speed when choosing the step size value. In attempts to optimise both the steady-state performance and convergence speed, techniques based on fuzzy logic (FL) tuning of LMS's step size have been developed [15-19]. An application of using FL-based step-size algorithm to blind source separation is given in [20]. The CMA is a stochastic gradient blind adaptive algorithm, and its step size has to be chosen with extreme care, much more so than the training-based LMS algorithm. While there exist some works on variable step-size CMA techniques [21,22], we are not aware of any published work on FL tuning of CMA's step size for blind equalisation, certainly not for application to high-order QAM blind equalisation, which is a much more difficult task than blind equalisation of binary phase shift keying or quadrature phase shift keying channels.

Against this background, this contribution investigates the fuzzy step-size CMA in the context of high-order QAM blind equalisation. Specifically, an FL tuning unit is designed to adjust the step size of the CMA. This fuzzy step-size CMA is also combined with the SDD scheme to obtain the concurrent FL assisted CMA and SDD blind equaliser. The benefits of using this FL-based step size approach are studied using simulation, and the results obtained show that the FL assisted CMA and SDD scheme achieves faster convergence over the constant step-size CMA and SDD scheme. The paper is organised as follows. Section 2 presents the channel signal model and the equalisation structure. In Section 3, the CMA as well as the concurrent CMA and SDD scheme are briefly summarised. This is followed with a detailed description of the FL assisted CMA. The concurrent FL assisted CMA and SDD blind equaliser is then introduced. Section 4 investigates the achievable performance of the CMA, the CMA and SDD, the FL assisted CMA as well as the FL-assisted CMA and SDD-based blind equalisers, using the performance of the MMSE equalisation solution given the perfect channel information as the benchmark. The paper concludes at Section 5 .

\section{Equalisation signal model}

Consider the frequency selective channel, whose symbol-rate channel impulse response $(\mathrm{CIR})$ is denoted by $\mathbf{c}_{\mathrm{CIR}}=$ $\left[c_{0} c_{1} \cdots c_{n_{\mathrm{ch}}-1}\right]^{T}$. Here, $n_{\mathrm{ch}}$ is the length of the CIR and $c_{i}, 0 \leqslant i \leqslant n_{\mathrm{ch}}-1$, are complex-valued CIR taps. The symbol-rate received signal sample $x(k)$ is expressed by [23]

$$
x(k)=\sum_{i=0}^{n_{\mathrm{ch}}-1} c_{i} s(k-i)+e(k),
$$

where $e(k)$ is a complex-valued additive white Gaussian noise with $E\left[|e(k)|^{2}\right]=2 \sigma_{e}^{2}$, and $s(k)$ is the $k$-th transmitted symbol with the symbol energy $E\left[|s(k)|^{2}\right]=\sigma_{s}^{2}$. The modulation scheme is assumed to be the $M$-QAM and therefore $s(k)$ takes the value from the symbol set

$$
\mathcal{S} \triangleq\left\{s_{i, l}=u_{i}+j u_{l}, 1 \leqslant i, l \leqslant \sqrt{M}\right\},
$$

where $j \triangleq \sqrt{-1}$, the real-part symbol $\Re\left[s_{i, l}\right]=u_{i}=2 i-\sqrt{M}-1$ and the imaginary-part symbol $\Im\left[s_{i, l}\right]=u_{l}=2 l-\sqrt{M}-1$. The channel signal-to-noise ratio (SNR) is defined as

$$
\mathrm{SNR} \triangleq\left(\sum_{i=0}^{n_{\mathrm{ch}}-1}\left|c_{i}\right|^{2} \sigma_{s}^{2}\right) / 2 \sigma_{e}^{2} .
$$

The equaliser has a length $n_{\mathrm{eq}}$, and its output is given by

$$
y(k)=\sum_{i=0}^{n_{\mathrm{eq}}-1} w_{i}^{*} x(k-i)=\mathbf{w}^{H} \mathbf{x}(k),
$$

where the equaliser's weight vector $\mathbf{w}=\left[w_{0} w_{1} \cdots w_{n_{\mathrm{eq}}-1}\right]^{T}$ and the channel observation vector $\mathbf{x}(k)=[x(k) x(k-1) \cdots x(k-$ $\left.\left.n_{\mathrm{eq}}+1\right)\right]^{T}$. The equaliser output $y(k)$ is passed to the decision device to produce an estimate $\hat{s}(k-\tau)$ of the transmitted 
symbol $s(k-\tau)$, where $0 \leqslant \tau \leqslant \tau_{\max } \triangleq n_{\mathrm{eq}}+n_{\mathrm{ch}}-2$ is the equaliser's decision delay. For the blind equalisation application, the decision delay $\tau$ is unknown.

It is straightforward to verify that the equaliser's input vector $\mathbf{x}(k)$ can be expressed as

$$
\mathbf{x}(k)=\mathbf{C s}(k)+\mathbf{e}(k),
$$

where the noise vector $\mathbf{e}(k)=\left[e(k) e(k-1) \cdots e\left(k-n_{\mathrm{eq}}+1\right)\right]^{T}$, the transmitted symbol vector $\mathbf{s}(k)=[s(k) s(k-1) \cdots s(k-$ $\left.\left.\tau_{\max }\right)\right]^{T}$ and the $n_{\mathrm{eq}} \times\left(\tau_{\max }+1\right)$ CIR matrix $\mathbf{C}$ has a Toeplitz form

$$
\mathbf{C} \triangleq\left[\begin{array}{ccccccc}
c_{0} & c_{1} & \cdots & c_{n_{\mathrm{ch}}-1} & 0 & \cdots & 0 \\
0 & c_{0} & c_{1} & \cdots & c_{n_{\mathrm{ch}}-1} & \ddots & \vdots \\
\vdots & \ddots & \ddots & \ddots & \cdots & \ddots & 0 \\
0 & \cdots & 0 & c_{0} & c_{1} & \cdots & c_{n_{\mathrm{ch}}-1}
\end{array}\right]=\left[\mathbf{c}_{0} \mathbf{c}_{1} \cdots \mathbf{c}_{\tau_{\max }}\right],
$$

with $\mathbf{c}_{i}, 0 \leqslant i \leqslant \tau_{\max }$, denoting the $i$-th column of $\mathbf{C}$. With the perfect channel information, the optimal MMSE equalisation solution that minimises the MSE $J_{\mathrm{MSE}}(\mathbf{w}) \triangleq E\left[|s(k-\tau)-y(k)|^{2}\right]$ is given by [14]

$$
\mathbf{w}_{\mathrm{MMSE}}=\left(\mathbf{C C}^{H}+\frac{2 \sigma_{e}^{2}}{\sigma_{s}^{2}} \mathbf{I}_{n_{\mathrm{eq}}}\right)^{-1} \mathbf{c}_{\tau},
$$

where $\mathbf{I}_{n_{\mathrm{eq}}}$ denotes the $n_{\mathrm{eq}} \times n_{\mathrm{eq}}$-dimensional identity matrix.

Before blind adaptation, the middle tap of the equaliser weight vector $\mathbf{w}(0)$ is initialised to $1+j 0$ and the rest of the weights are set to $0+j 0$. For the equaliser with the weight vector $\mathbf{w}$ and a (unknown) decision delay $\tau$, define the combined equaliser and channel impulse response as

$$
\mathbf{f}^{T}=\left[f_{0} f_{1} \cdots f_{\tau_{\max }}\right] \triangleq \mathbf{w}^{H} \mathbf{C}
$$

and let

$$
i_{\max }=\arg \max _{0 \leqslant i \leqslant \tau_{\max }}\left|f_{i}\right|
$$

The equaliser's decision delay is in fact $\tau=i_{\text {max }}$. In simulation, the quality of equalisation can be judged using the maximum distortion (MD) measure defined by

$$
\mathrm{MD}(\mathbf{w}) \triangleq\left(\sum_{i=0}^{\tau_{\max }}\left|f_{i}\right|-\left|f_{i_{\max }}\right|\right) /\left|f_{i_{\max }}\right| .
$$

Alternatively, the equalisation performance can be assessed using the MSE criterion given by

$$
J_{\mathrm{MSE}}(\mathbf{w}) \triangleq \sigma_{s}^{2}\left(\left(1-\mathbf{w}^{H} \mathbf{c}_{\tau}-\mathbf{w}^{T} \mathbf{c}_{\tau}^{*}\right)+\mathbf{w}^{H}\left(\mathbf{C C}^{H}+\frac{2 \sigma_{e}^{2}}{\sigma_{s}^{2}} \mathbf{I}_{n_{\mathrm{eq}}}\right) \mathbf{w}\right) .
$$

Ultimately, the SER can be simulated to assess the equalisation performance.

\section{Blind equalisation algorithms}

\subsection{Constant modulus algorithm}

At the sample $k$, given the equaliser output $y(k)=\mathbf{w}^{H}(k-1) \mathbf{x}(k)$, the CMA adapts the equaliser's weight vector $\mathbf{w}$ according to $[2,3]$

$$
\left\{\begin{array}{l}
\varepsilon(k)=y(k)\left(\Delta-|y(k)|^{2}\right), \\
\mathbf{w}(k)=\mathbf{w}(k-1)+\mu_{\mathrm{CMA}} \varepsilon^{*}(k) \mathbf{x}(k),
\end{array}\right.
$$

where $\Delta=E\left[|s(k)|^{4}\right] / E\left[|s(k)|^{2}\right]$ and $\mu_{\mathrm{CMA}}$ is the step size of the CMA. Typically, a very small $\mu_{\mathrm{CMA}}$ has to be used to ensure convergence. The CMA is known to be very robust, and the standard convergence analysis of the CMA can be found for example in [6]. The computational complexity of this CMA is summarised in Table 1. 
Table 1

Computational requirements per weight update, where $n_{\mathrm{eq}}$ is the length of the equaliser and the symbol rate is $N_{\mathrm{sm}}$ times faster than the operational rate of the FIS.

\begin{tabular}{lll}
\hline Algorithm & Multiplications & Additions \\
\hline CMA & $8 \times n_{\mathrm{eq}}+6$ & $8 \times n_{\mathrm{eq}}$ \\
CMA + SDD & $12 \times n_{\mathrm{eq}}+29$ & $14 \times n_{\mathrm{eq}}+21$ \\
FIS & $2+22 / N_{\mathrm{sm}}$ & $2+22 / N_{\mathrm{sm}}$ \\
\hline
\end{tabular}

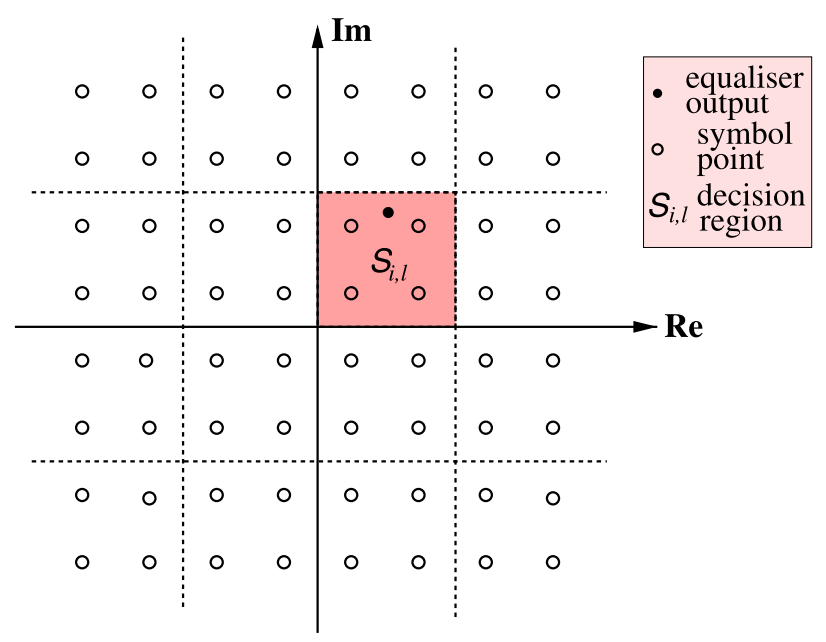

Fig. 1. Local decision region partition for soft decision-directed adaptation with 64-QAM constellation.

\subsection{Combined CMA and SDD scheme}

Let the equaliser's weight vector be split into two parts, yielding $\mathbf{w}=\mathbf{w}_{c}+\mathbf{w}_{d}$. The initial $\mathbf{w}_{c}$ and $\mathbf{w}_{d}$ are simply set to $\mathbf{w}_{c}(0)=\mathbf{w}_{d}(0)=0.5 \mathbf{w}(0)$. In particular, the weight vector $\mathbf{w}_{c}$ is updated using the CMA of (12) by substituting $\mathbf{w}_{c}$ in the place of $\mathbf{w}$. The weight vector $\mathbf{w}_{d}$ by contrast is updated using the SDD scheme [10-12], which has its root in the blind equalisation scheme of [13]. Specifically, the complex phasor plane is divided into the $M / 4$ square or rectangular regions, as illustrated in Fig. 1, and each region $\mathcal{S}_{i, l}$ contains four symbol points as defined by

$$
\mathcal{S}_{i, l}=\left\{s_{r, m}, r=2 i-1,2 i, m=2 l-1,2 l\right\},
$$

where $1 \leqslant i, l \leqslant \sqrt{M} / 2$. If the equaliser's output $y(k) \in \mathcal{S}_{i, l}$, a local approximation of the marginal probability density function (PDF) of $y(k)$ is given by [10-12]

$$
\hat{p}(\mathbf{w}, y(k)) \approx \sum_{r=2 i-1}^{2 i} \sum_{m=2 l-1}^{2 l} \frac{1}{8 \pi \rho} e^{-\frac{\left|y(k)-s_{r, m}\right|^{2}}{2 \rho}},
$$

where $\rho$ defines the cluster width associated with the four clusters of each region $\mathcal{S}_{i, l}$. The SDD algorithm is designed to maximise the $\log$ of the local marginal PDF criterion $E\left[J_{\operatorname{LMAP}}(\mathbf{w}, y(k))\right]$, where $J_{\operatorname{LMAP}}(\mathbf{w}, y(k))=\rho \log (\hat{p}(\mathbf{w}, y(k))), \mathrm{using}$ a stochastic gradient optimisation. That is, $\mathbf{w}_{d}$ is updated according to [10-12]

$$
\mathbf{w}_{d}(k)=\mathbf{w}_{d}(k-1)+\mu_{\mathrm{SDD}} \frac{\partial J_{\mathrm{LMAP}}(\mathbf{w}(k-1), y(k))}{\partial \mathbf{w}_{d}},
$$

where $\mu_{\mathrm{SDD}}$ is the step size of the SDD, and

$$
\frac{\partial J_{\mathrm{LMAP}}(\mathbf{w}, y(k))}{\partial \mathbf{w}_{d}}=\frac{1}{Z_{N}} \sum_{r=2 i-1}^{2 i} \sum_{m=2 l-1}^{2 l} e^{-\frac{\mid y(k)-s_{r}, m}{2 \rho}}\left(s_{r, m}-y(k)\right)^{*} \mathbf{x}(k),
$$

with the normalisation factor

$$
Z_{N}=\sum_{r=2 i-1}^{2 i} \sum_{m=2 l-1}^{2 l} e^{-\frac{\left|y(k)-s_{r, m}\right|^{2}}{2 \rho}}
$$




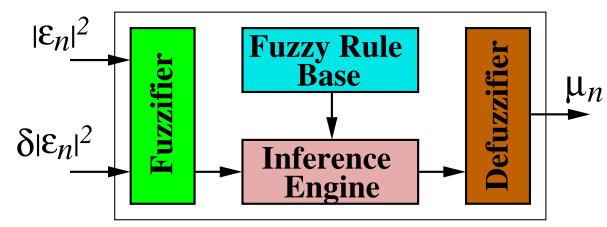

Fig. 2. Schematic of the fuzzy inference system for tuning the step size of the CMA. The operational rate $n$ of the FIS is $N_{\mathrm{sm}}$ times slower than the symbol rate $k$.

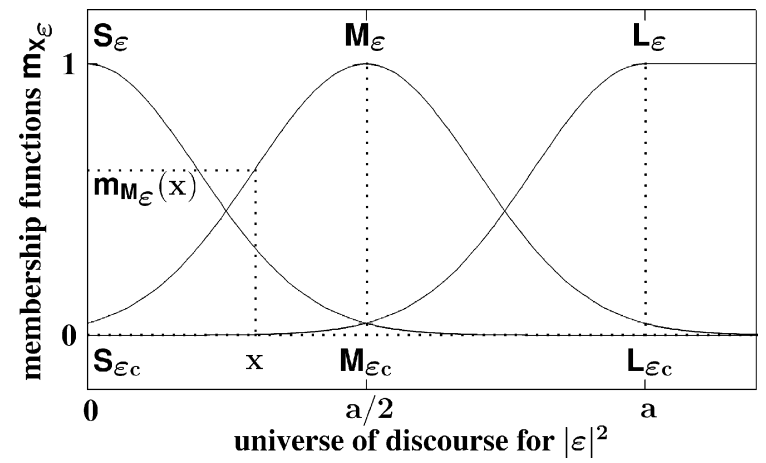

Fig. 3. Membership functions over the universe of discourse for $\left|\varepsilon_{n}\right|^{2}$, where the fuzzy variable $X=S$, $M$ or $L$.

The computational complexity of this combined CMA and SDD scheme (CMA + SDD) is also given in Table 1. The choice of the cluster width $\rho$, defined in the context of the local PDF (14), should ensure a proper separation of the four clusters of $\mathcal{S}_{i, l}$ [13]. As the minimum distance between the two neighbouring constellation points is $2, \rho$ is typically chosen to be less than 1 . The performance of the algorithm is not overly sensitive to the value of $\rho$ employed and an appropriate $\rho$ can easily be chosen from a large range of values. More specifically, when the objective of equalisation is accomplished, $y(k) \approx s(k-\tau)+\hat{e}(k)$, where $\hat{e}(k)$ is Gaussian distributed with zero mean. Therefore, the value of $\rho$ is related to the variance of $\hat{e}(k)$, which is $2 \sigma_{e}^{2} \mathbf{w}^{H} \mathbf{w}$. Thus, for high SNR situations, small $\rho$ can be used, while for low SNR cases, large $\rho$ is preferred. Soft decision nature becomes explicit in (16), because rather than committing to a single hard decision $\mathcal{Q}[y(k)]$, where $\mathcal{Q}[\bullet]$ denotes the quantisation operator, as the hard DD scheme would, alternative decisions are also considered in the local region $\mathcal{S}_{i, l}$ that includes $\mathcal{Q}[y(k)]$, and each tentative decision is weighted by an exponential term $e^{\{\bullet\}}$, which is a function of the distance between the equaliser's soft output $y(k)$ and the tentative decision $s_{r, m}$. This soft decision nature substantially reduces the risk of error propagation and achieves faster convergence, compared with the hard DD scheme [10-12].

\subsection{Fuzzy step-size CMA}

For the fuzzy step-size CMA, we choose the fuzzy inference system (FIS) of Fig. 2, which maps the two input variables, $\left|\varepsilon_{n}\right|^{2}$ and $\delta\left|\varepsilon_{n}\right|^{2}$, into an appropriate step size $\mu_{n}$. The operation of the FIS is based on the principle of fuzzy logic [24,25]. The two input variables are defined respectively as

$$
\begin{aligned}
& \left|\varepsilon_{n}\right|^{2}=\frac{1}{N_{\mathrm{sm}}} \sum_{l=0}^{N_{\mathrm{sm}}-1}|\varepsilon(k-l)|^{2}, \\
& \delta\left|\varepsilon_{n}\right|^{2}=\left|\varepsilon_{n}\right|^{2}-\left|\varepsilon_{n-1}\right|^{2},
\end{aligned}
$$

where $n=\left\lfloor k / N_{\mathrm{sm}}\right\rfloor$ with $\lfloor\bullet\rfloor$ denoting the integer floor operator, and $N_{\mathrm{sm}}$ is the short-term average length. Note that the FIS operates once every $N_{\mathrm{sm}}$ samples, and the output $\mu_{n}$ is used as the step size of the CMA for the subsequent $N_{\mathrm{sm}}$ samples, namely,

$$
\mu_{\mathrm{CMA}}=\mu_{n}, \quad n \cdot N_{\mathrm{sm}} \leqslant k<(n+1) \cdot N_{\mathrm{sm}} .
$$

The required initial conditions can be set to $\left|\varepsilon_{0}\right|^{2}=0$ and $\mu_{0}=\mu_{\min }$, where $\mu_{\min }$ represents the smallest value for the step size.

The two crisp input variables are transformed separately to the respective degrees, to which they belong to the corresponding fuzzy sets via appropriate membership functions (MBFs). The fuzzy sets used to partition the universe of discourse for $\left|\varepsilon_{n}\right|^{2}$ are labelled as small $\left(\mathrm{S}_{\varepsilon}\right)$, medium $\left(\mathrm{M}_{\varepsilon}\right)$ and large $\left(\mathrm{L}_{\varepsilon}\right)$, and their associate MBFs are shown in Fig. 3, where $\mathrm{S}_{\varepsilon c}$, $\mathrm{M}_{\varepsilon c}$ and $\mathrm{L}_{\varepsilon c}$ are the centroids of $\mathrm{S}_{\varepsilon}, \mathrm{M}_{\varepsilon}$ and $\mathrm{L}_{\varepsilon}$, respectively. The Gaussian MBFs

$$
\mathrm{m}_{\mathbf{X}_{\varepsilon}}(x)=e^{-\frac{\left(x-X_{\varepsilon c}\right)^{2}}{2 \rho_{\varepsilon}}}
$$




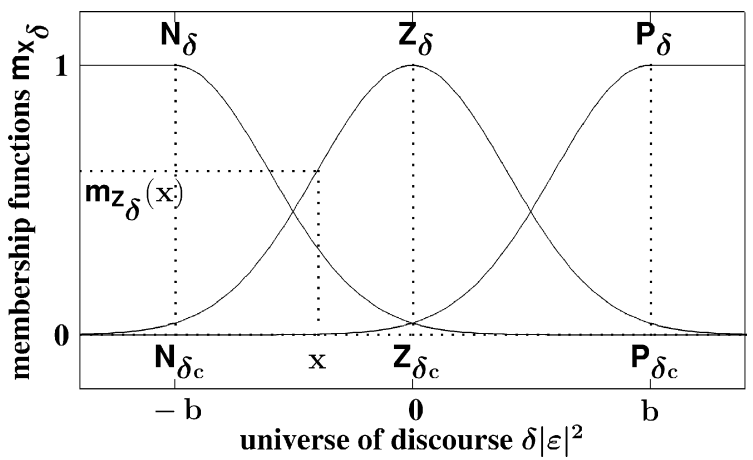

Fig. 4. Membership functions over the universe of discourse for $\delta\left|\varepsilon_{n}\right|^{2}$, where the fuzzy variable $X=N, Z$ or $P$.

Table 2

Fuzzy sets for crisp $\mu_{n}$.

\begin{tabular}{llll}
\hline Fuzzy set & $\mathrm{S}_{\mu}$ & $\mathrm{M}_{\mu}$ & $\mathrm{L}_{\mu}$ \\
\hline Centroid & $\mu_{\min }$ & $2 \mu_{\min }$ & $\mu_{\max }=4 \mu_{\min }$ \\
Universe of discourse & & {$\left[\mu_{\min }, \mu_{\max }\right]$} & \\
\hline
\end{tabular}

\begin{tabular}{|c|c|c|c|c|}
\hline \multirow{2}{*}{\multicolumn{2}{|c|}{$\mu_{n}$}} & \multicolumn{3}{|c|}{$\delta\left|\varepsilon_{n}\right|^{2}$} \\
\hline & & $\mathrm{N}_{\delta}$ & $\mathrm{Z}_{\delta}$ & $\mathrm{P}_{\delta}$ \\
\hline \multirow{3}{*}{$\frac{\bar{w}}{\underline{w}}$} & $\mathrm{~S}_{\varepsilon}$ & ${ }^{1} L_{\mu}$ & ${ }^{2} M_{\mu}$ & ${ }^{3} S_{\mu}$ \\
\hline & $M_{\varepsilon}$ & ${ }^{4} M_{\mu}$ & $5 S_{\mu}$ & ${ }^{6} S_{\mu}$ \\
\hline & $L_{\varepsilon}$ & ${ }^{7} \mathrm{M}_{\mu}$ & ${ }^{8} S_{\mu}$ & ${ }^{9} \mathrm{~S}_{\mu}$ \\
\hline
\end{tabular}

Fig. 5. Fuzzy rule table for the step size $\mu_{n}$.

are used in this study, where $X_{\varepsilon}$ represents $S_{\varepsilon}, M_{\varepsilon}$ or $L_{\varepsilon}$, with the exception that $m_{L_{\varepsilon}}(x)=1$ for $x \geqslant a$. Similarly, the fuzzy sets used to partition the universe of discourse for $\delta\left|\varepsilon_{n}\right|^{2}$ are labelled as negative $\left(\mathrm{N}_{\delta}\right)$, zero $\left(\mathrm{Z}_{\delta}\right)$ and positive $\left(\mathrm{P}_{\delta}\right)$, with the related MBFs shown in Fig. 4, where $\mathrm{N}_{\delta c}, \mathrm{Z}_{\delta c}$ and $\mathrm{P}_{\delta c}$ are the centroids of $\mathrm{N}_{\delta}, \mathrm{Z}_{\delta}$ and $\mathrm{P}_{\delta}$, respectively. Again the Gaussian MBFs

$$
\mathrm{m}_{\mathrm{X}_{\delta}}(x)=e^{-\frac{\left(x-\mathrm{x}_{\delta c}\right)^{2}}{2 \rho_{\delta}}}
$$

are used, where $\mathrm{X}_{\delta}$ represents $\mathrm{N}_{\delta}, \mathrm{Z}_{\delta}$ or $\mathrm{P}_{\delta}$. But we have $\mathrm{m}_{\mathrm{N}_{\delta}}(x)=1$ for $x \leqslant-b$ and $\mathrm{m}_{\mathrm{P}_{\delta}}(x)=1$ for $x \geqslant b$.

The universe of discourse for the step size $\mu_{n}$ is defined by $\left[\mu_{\min }, \mu_{\max }\right]$, and the fuzzy sets used to partition it are labelled as small $\left(S_{\mu}\right)$, medium $\left(M_{\mu}\right)$ and large $\left(L_{\mu}\right)$, as is summarised in Table 2. The fuzzy inference engine constructs a set of fuzzy IF-THEN rules. Since there are 3 fuzzy sets for each of $\left|\varepsilon_{n}\right|^{2}$ and $\delta\left|\varepsilon_{n}\right|^{2}$, the number of fuzzy IF-THEN rules is 9. These fuzzy IF-THEN rules are shown in Fig. 5. Rule 1, for example, reads like: IF $\left|\varepsilon_{n}\right|^{2}$ is $\mathrm{S}_{\varepsilon}$ AND $\delta\left|\varepsilon_{n}\right|^{2}$ is $\mathrm{N}_{\delta}$ THEN $\mu_{n}$ is $L_{\mu}$. Let $\mathrm{m}_{\mathrm{X}_{\mu}}\left(\mu_{n}[i]\right)$ be the MBF value at location $\mu_{n}[i]$, where $1 \leqslant i \leqslant 9$. The 9 locations $\mu_{n}[i], 1 \leqslant i \leqslant 9$, are specified by Fig. 5 and Table 2. For example, from Fig. 5 and Table 2, we have $\mu_{n}[1]=\mu_{\max }$. The min operator is applied to truncate the output fuzzy set for each rule. For instance, according to the fuzzy rule table of Fig. 5 , the MBF value at $\mu_{n}[1]$ is

$$
\mathrm{m}_{\mathrm{x}_{\mu}}\left(\mu_{n}[1]\right)=\min \left\{\mathrm{m}_{\mathrm{S}_{\varepsilon}}\left(\left|\varepsilon_{n}\right|^{2}\right), \mathrm{m}_{\mathrm{N}_{\delta}}\left(\delta\left|\varepsilon_{n}\right|^{2}\right)\right\}
$$

and so on. The defuzzification method used to obtain a crisp value for the step size is the following centroid calculation

$$
\mu_{n}=\frac{\sum_{i=1}^{9} \mu_{n}[i] \cdot \mathrm{m}_{\mu}\left(\mu_{n}[i]\right)}{\sum_{i=1}^{9} \mathrm{~m}_{\mathrm{x}_{\mu}}\left(\mu_{n}[i]\right)}
$$

which returns the centre of area under the aggregated MBF curve.

The extra computational complexity imposed by this FIS is given in Table 1. Suitable values for the short-term average length, $N_{s m}$, can typically be chosen in the range of 10 to 20 . The range of $\left|\varepsilon_{n}\right|^{2}$ is simply $a \approx \max |\varepsilon(k)|^{2}$, and our experience suggests that the variance of the Gaussian MBFs for $\left|\varepsilon_{n}\right|^{2}$ can be set to $\rho_{\varepsilon}=(0.01 a)^{2}$. For better efficiency, $\mathrm{P}_{\delta c}$ should be relatively small, and we find by experiment that $b=0.01 a$ to $0.001 a$ are appropriate depending on the size of QAM 


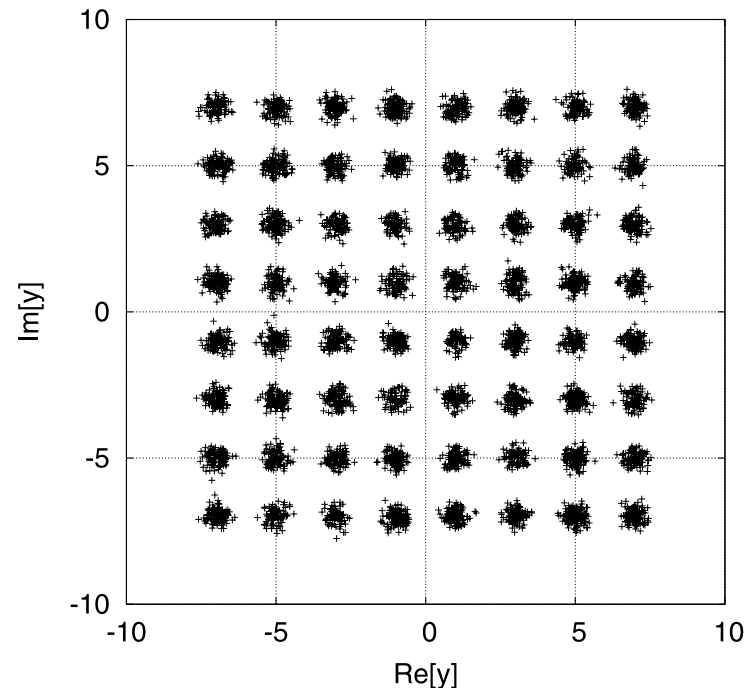

(a)

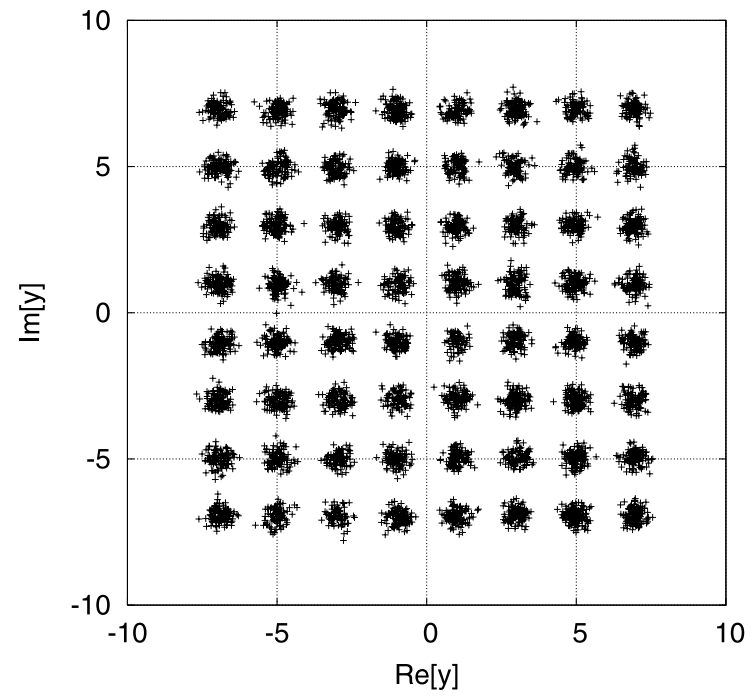

(c)

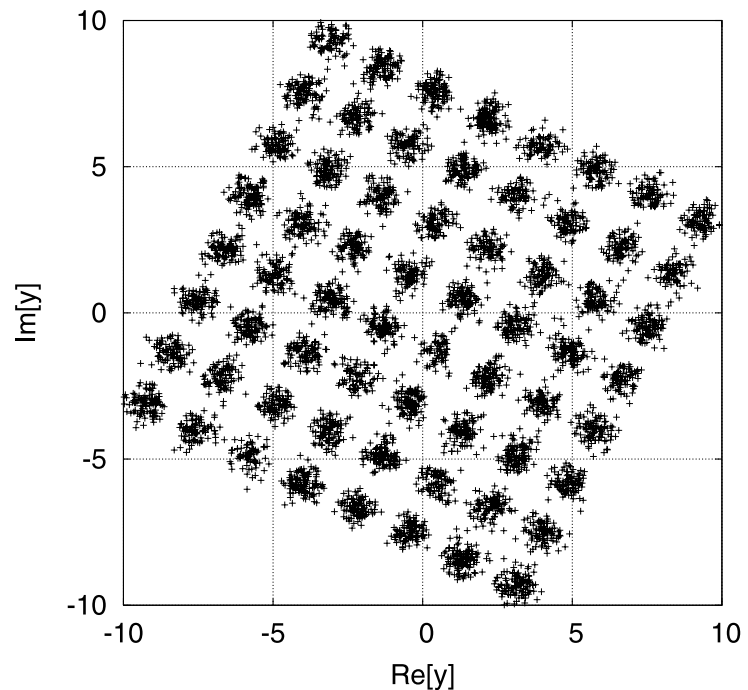

(b)

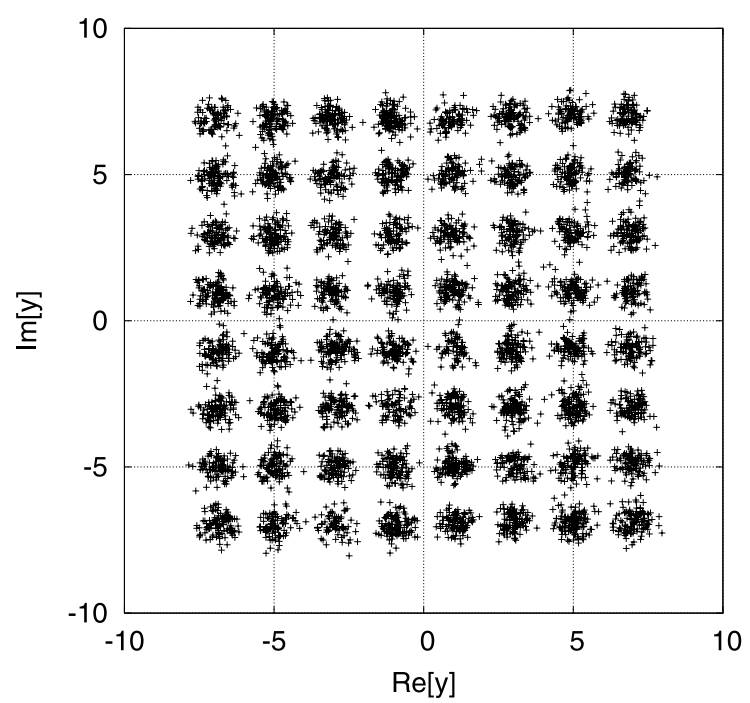

(d)

Fig. 6. Output constellation: (a) the MMSE equaliser, (b) the CMA equaliser, (c) the CMA + SDD equaliser, and (d) the CMA equaliser with $-27^{\circ}$ phase compensation, for the 64-QAM example with the channel length $n_{\mathrm{ch}}=5$, equaliser length $n_{\mathrm{eq}}=23$ and SNR $=38 \mathrm{~dB}$.

constellation $M$. The variance of the Gaussian MBFs for $\delta\left|\varepsilon_{n}\right|^{2}$ can be set to $\rho_{\delta}=(0.2 b)^{2}$. The minimum value of the step size $\mu_{\min }$ is simply chosen to be the value for the constant step-size CMA which produces satisfactory performance in terms of both steady-state error and convergence speed. These choices of the FIS's parameters have been verified by extensive simulation study.

\subsection{Combined fuzzy step-size CMA and SDD scheme}

The above fuzzy step-size CMA (FL-CMA) can be combined with the SDD adaptation to provide the concurrent fuzzy step-size CMA and SDD scheme (FL-CMA + SDD). Note that it is not necessary to adopt a variable step size strategy for the SDD adaptation, since the "error" or the stochastic gradient used for correcting the weights is well "normalised" by the normalisation factor $Z_{N}$ of (17).

\subsection{Alternative partition of blind equaliser}

In the above derivation of the concurrent CMA and SDD blind equaliser, we adopt the weight vector partion of $\mathbf{w}_{c}=$ $\mathbf{w}_{d}=0.5 \mathbf{w}$. A more generic partition is 


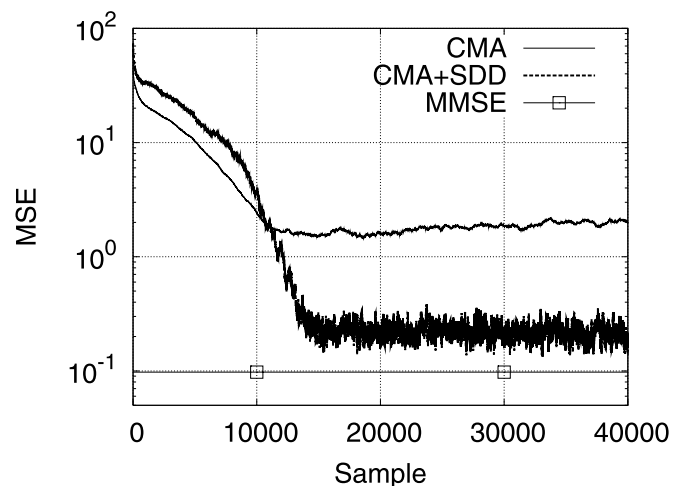

(a)

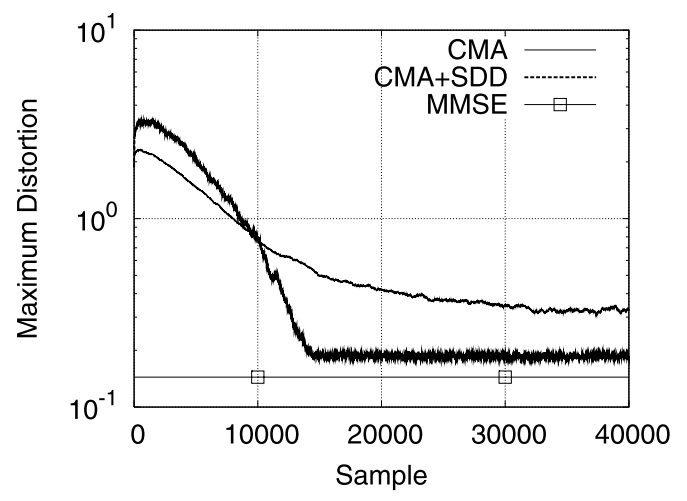

(b)

Fig. 7. Convergence performance comparison of the CMA and CMA + SDD, in terms of: (a) the MSE and (b) MD measure, averaged over 10 runs, for the 64-QAM example with $n_{\mathrm{ch}}=5, n_{\mathrm{eq}}=23$ and SNR $=38 \mathrm{~dB}$. The step size of the CMA is $\mu_{\mathrm{CMA}}=2 \times 10^{-7}$, while the step size and the cluster width of the $\mathrm{CMA}+\mathrm{SDD}$ are $\mu_{\mathrm{SDD}}=2 \times 10^{-4}$ and $\rho=0.6$.

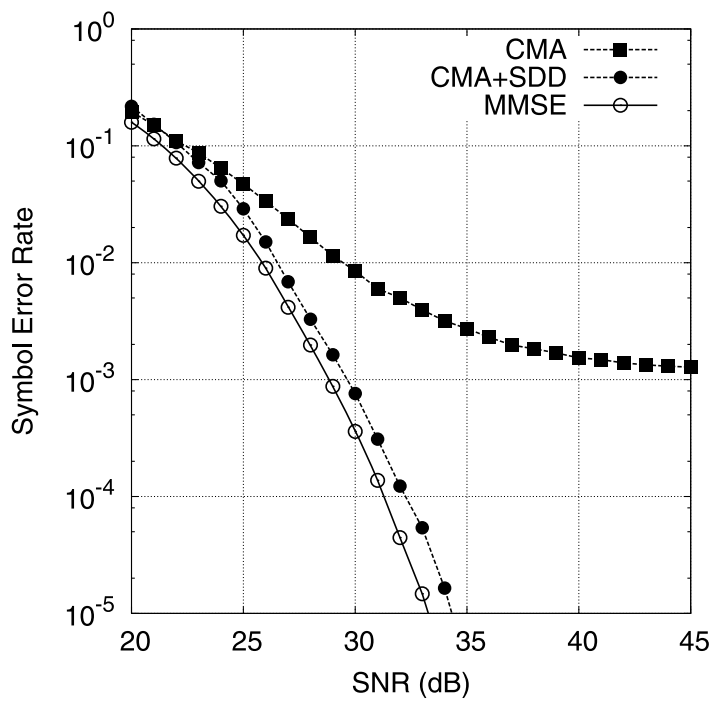

Fig. 8. Symbol error rate comparison of the three equalisers for the 64-QAM example with $n_{\mathrm{ch}}=5$ and $n_{\mathrm{eq}}=23$.

$$
\mathbf{w}=\alpha \mathbf{w}_{c}+(1-\alpha) \mathbf{w}_{d},
$$

where $0 \leqslant \alpha \leqslant 1$. It is clear that $\alpha=1$ is corresponding to a pure CMA blind equaliser while $\alpha=0$ is related to a pure SDD blind equaliser. Depending on the channel condition, appropriate value of $\alpha$ may be chosen to yield a potentially better equalisation performance. However, this appropriate weight value can be difficult to find.

\section{Simulation study}

\subsection{4-QAM example}

For this example, the modulation scheme was 64-QAM, the channel length was $n_{\mathrm{ch}}=5$ and the CIR $\mathbf{c}_{\mathrm{CIR}}$ was given by

$$
[-0.2+j 0.3-0.5+j 0.40 .7-j 0.60 .4+j 0.30 .2+j 0.1]^{T} .
$$

The equaliser length was chosen to be $n_{\mathrm{eq}}=23$. With $\mathbf{w}(0)$ initialised to all zero elements except the middle tap to $1+j 0$, the actual decision delay of the blind equaliser was $\tau=13$. Given SNR $=38 \mathrm{~dB}$, Fig. 6(a) shows the equaliser output constellation for the MMSE equaliser with $\tau=13$, while Figs. 6(b) and 6(c) depict the equaliser output constellations for the CMA and CMA + SDD blind equalisers, respectively, after convergence. The appropriate step size of the CMA was found empirically to be $\mu_{\mathrm{CMA}}=2 \times 10^{-7}$, while $\mu_{\mathrm{SDD}}=2 \times 10^{-4}$ and $\rho=0.6$ were found appropriate for the CMA + SDD. From Fig. 6(b), it can be seen that the CMA introduced $-27^{\circ}$ phase rotation. With a $-27^{\circ}$ phase compensation, the output constellation of the converged CMA blind equaliser is re-plotted in Fig. 6(d). The learning curves of the blind CMA and 


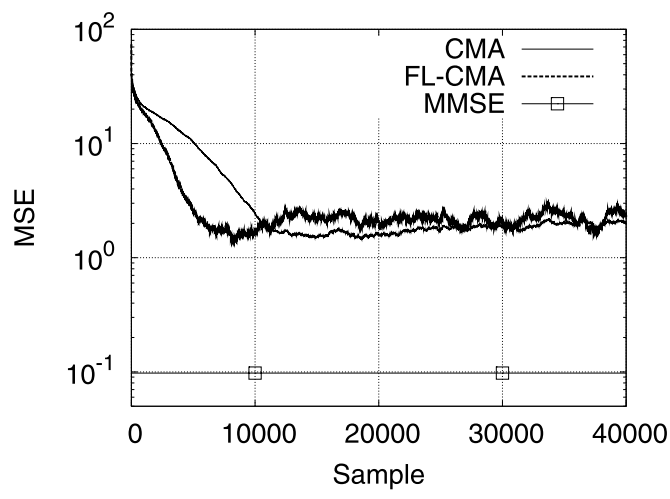

(a)

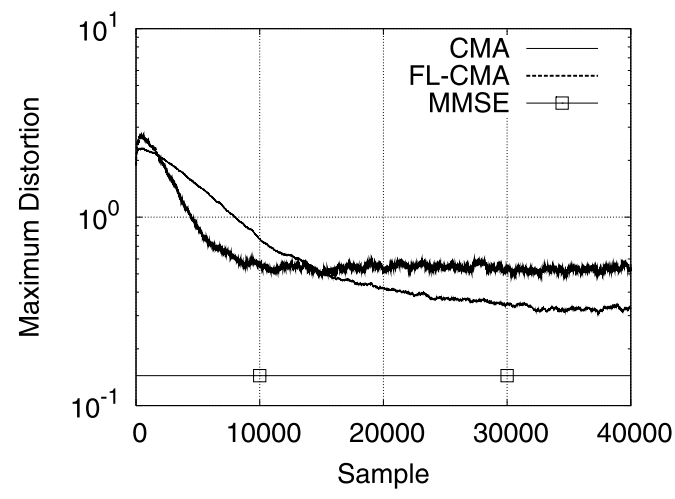

(b)

Fig. 9. Convergence performance comparison of the CMA and FL-CMA, in terms of: (a) the MSE and (b) MD measure, averaged over 10 runs, for the 64-QAM example with $n_{\mathrm{ch}}=5, n_{\mathrm{eq}}=23$ and $\mathrm{SNR}=38 \mathrm{~dB}$.

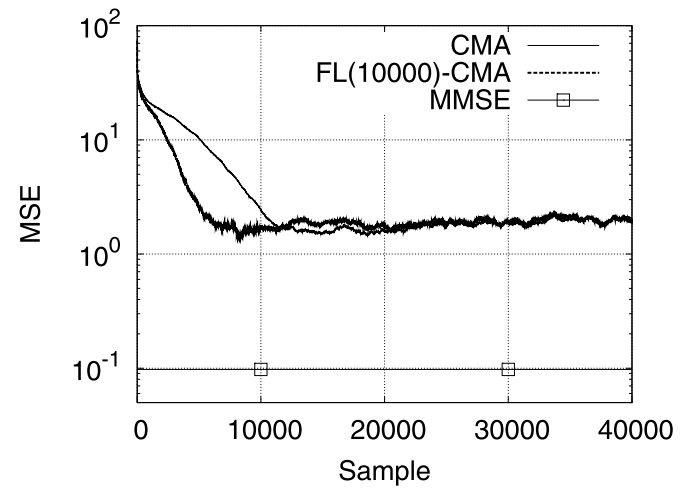

(a)

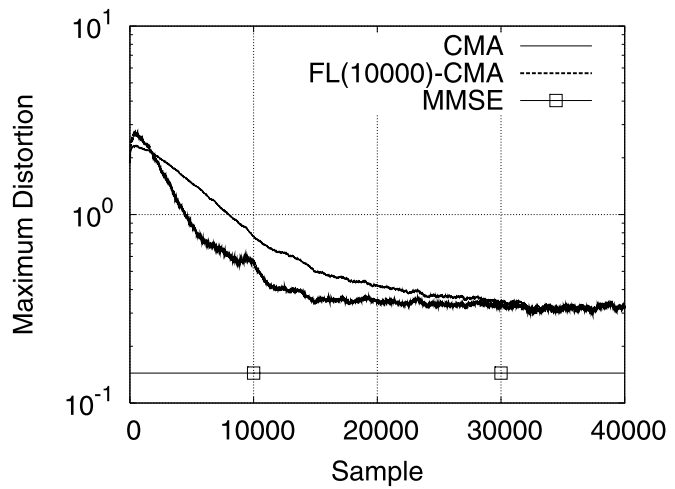

(b)

Fig. 10. Convergence performance comparison of the CMA and FL(10,000)-CMA, in terms of: (a) the MSE and (b) MD measure, averaged over 10 runs, for the 64-QAM example with $n_{\mathrm{ch}}=5, n_{\mathrm{eq}}=23$ and SNR $=38 \mathrm{~dB}$. The FL(10,000)-CMA uses the fuzzy step size for the first 10,000 samples and then switches to a constant step size.

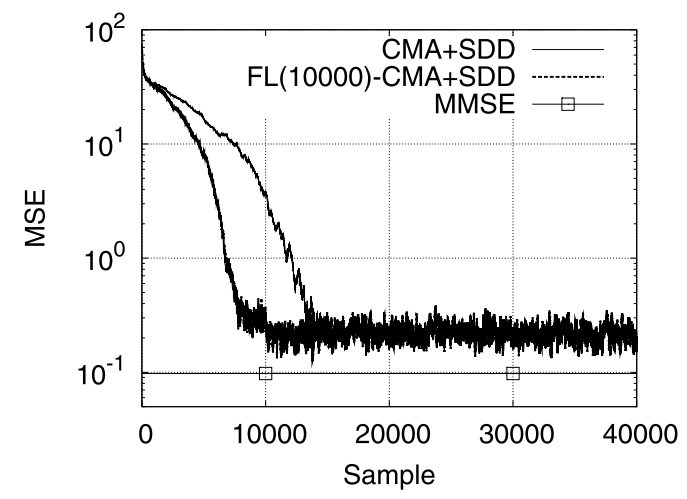

(a)

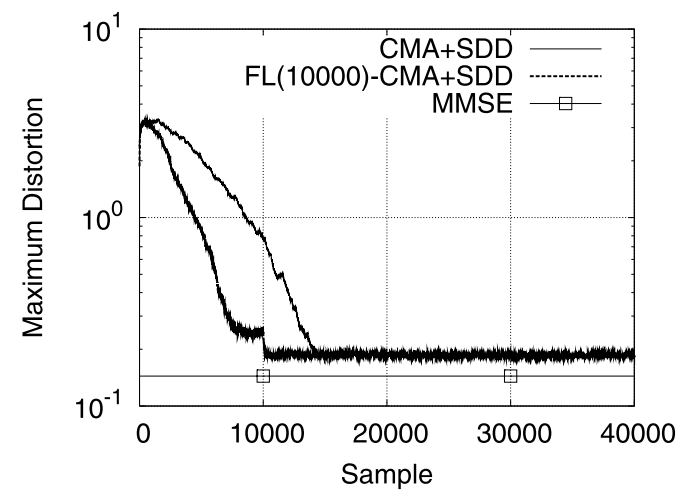

(b)

Fig. 11. Convergence performance comparison of the CMA + SDD and FL(10,000)-CMA + SDD, in terms of: (a) the MSE and (b) MD measure, averaged over 10 runs, for the 64-QAM example with $n_{\mathrm{ch}}=5, n_{\mathrm{eq}}=23$ and SNR $=38 \mathrm{~dB}$. The $\mathrm{FL}(10,000)$-CMA uses the fuzzy step size for the first 10,000 samples and then switches to a constant step size.

CMA + SDD equalisers, averaged over 10 runs and quantified in terms of the MSE as well as MD measures, are depicted in Fig. 7 with the MMSE solution as the benchmark, where the MSE of the CMA was calculated with the $-27^{\circ}$ phase compensation. The SER performance of the three equalisers, namely, the MMSE, the CMA and the CMA+SDD, are compared in Fig. 8, where again the CMA had the $-27^{\circ}$ phase compensation. 


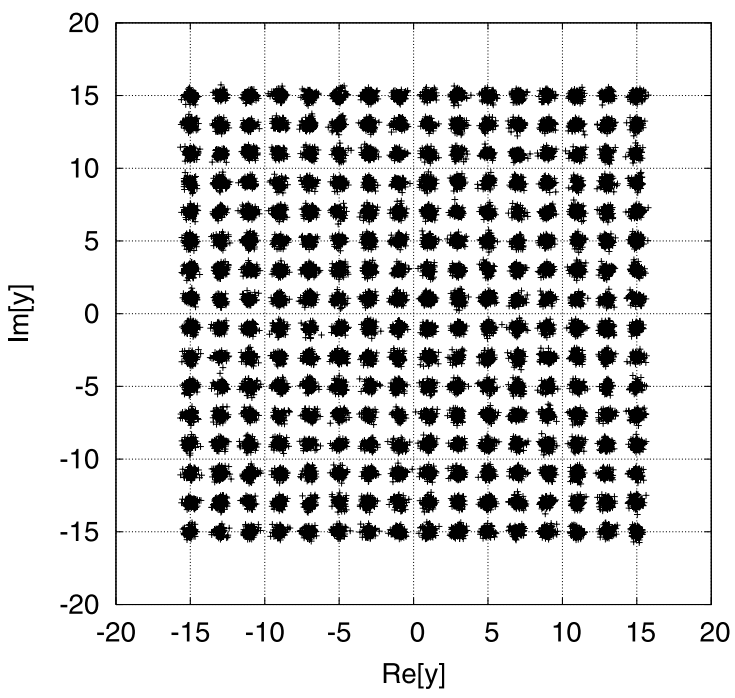

(a)

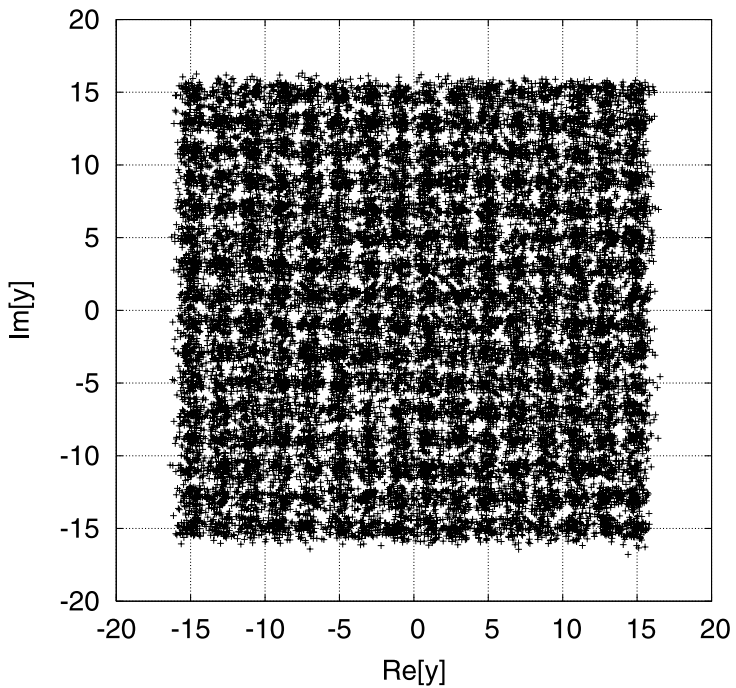

(b)

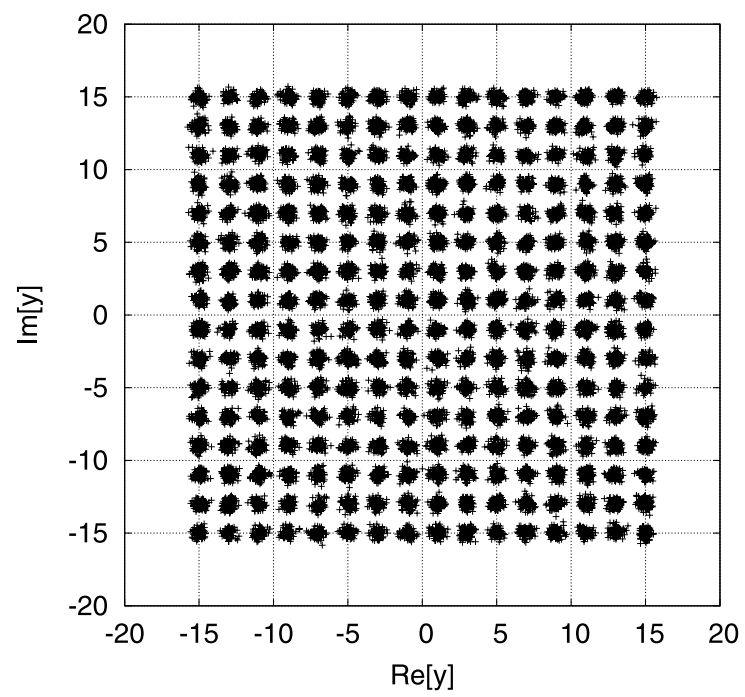

(c)

Fig. 12. Output constellation: (a) the MMSE equaliser, (b) the CMA equaliser with $-29^{\circ}$ phase compensation, and (c) the CMA + SDD equaliser, for the 256-QAM example with the channel length $n_{\mathrm{ch}}=3$, equaliser length $n_{\mathrm{eq}}=15$ and $\mathrm{SNR}=40 \mathrm{~dB}$.

The FL tuning unit for the step size of the CMA was next investigated. For 64-QAM

$$
\max |\varepsilon(k)|^{2} \approx \max \left\{|s(k)|^{2}\left(\Delta-|s(k)|^{2}\right)^{2}\right\} \approx 10^{5},
$$

and, therefore, we set the centroid of $\mathrm{L}_{\varepsilon}$ to $a=10^{5}$ and chose $b=0.01 a=10^{3}$ as the centroid of $\mathrm{P}_{\delta}$. The variances of the Gaussian MBFs were set to $\rho_{\varepsilon}=(0.01 a)^{2}$ and $\rho_{\delta}=(0.2 b)^{2}$ for $\left|\varepsilon_{n}\right|^{2}$ and $\delta\left|\varepsilon_{n}\right|^{2}$, respectively. The short-term average length for calculating $\left|\varepsilon_{n}\right|^{2}$ was chosen to be $N_{\mathrm{sm}}=20$, while $\mu_{\mathrm{min}}=2 \times 10^{-7}$ was adopted as the smallest value for $\mu_{n}$. Given SNR $=38 \mathrm{~dB}$, the convergence performance of this fuzzy step-size CMA, labelled as the FL-CMA, is compared with that of the CMA with a constant step size $\mu_{\mathrm{CMA}}=2 \times 10^{-7}$ in Fig. 9, where it can be seen that this FL-CMA did achieve a significantly faster convergence. However, its steady-state performance was poorer than the CMA, since the step size of the FL-CMA was always larger than or equal to $2 \times 10^{-7}$.

It was not difficult to re-design the parameters of the FL tuning unit so that the resulting FL-CMA could achieve the same steady-state performance as the CMA but the gain in convergence speed would somewhat diminish. A better strategy is to use this FL-CMA in the initial stage of blind adaptation for the maximum benefit in convergence rate and then to switch to the constant step-size CMA for the same good steady-state performance. Fig. 10 shows learning curve of this switched FL-CMA, labelled as the FL(10,000)-CMA, where the FL-CMA was used for the initial adaptation of 10,000 samples and the CMA of a constant step size $\mu_{\mathrm{CMA}}=2 \times 10^{-7}$ was used afterward. The choice of 10,000 was based on the observation 


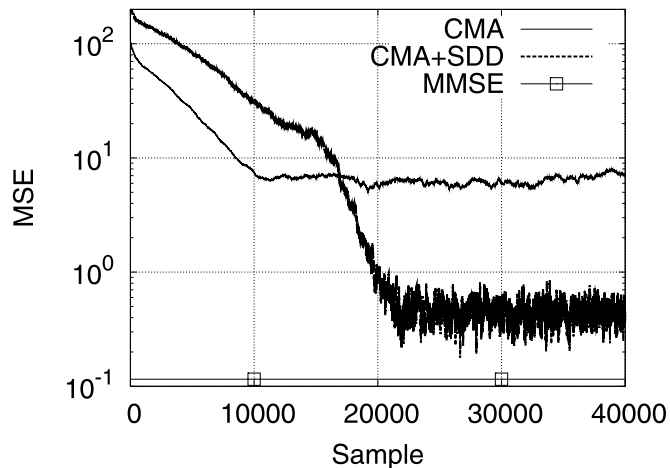

(a)

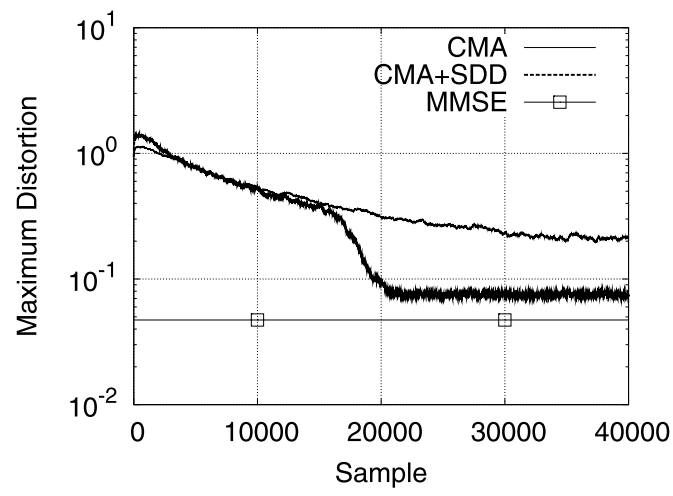

(b)

Fig. 13. Convergence performance comparison of the CMA and CMA + SDD, in terms of: (a) the MSE and (b) MD measure, averaged over 10 runs, for the 256-QAM example with $n_{\mathrm{ch}}=3, n_{\mathrm{eq}}=15$ and $\mathrm{SNR}=40 \mathrm{~dB}$. The step size of the CMA is $\mu_{\mathrm{CMA}}=2 \times 10^{-8}$, while the step size and the cluster width of the $\mathrm{CMA}+\mathrm{SDD}$ are $\mu_{\mathrm{SDD}}=6 \times 10^{-5}$ and $\rho=0.4$.

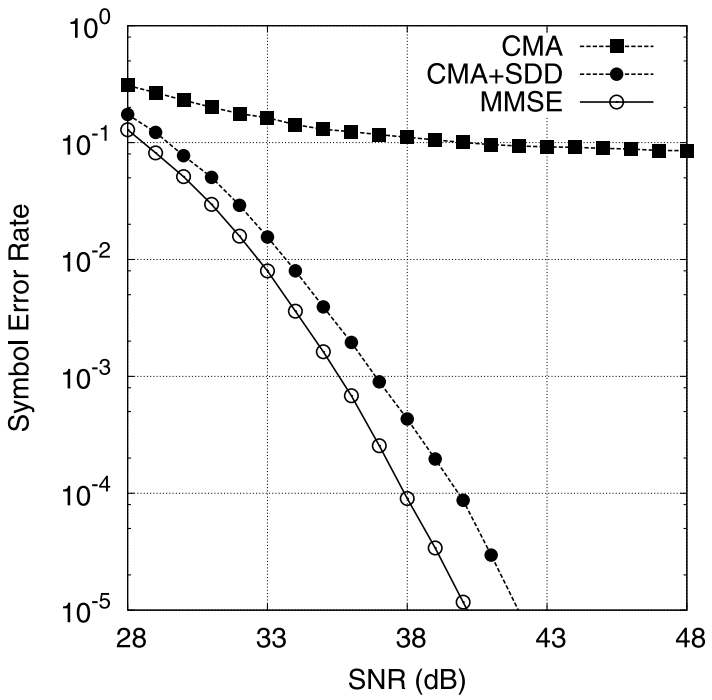

Fig. 14. Symbol error rate comparison of the three equalisers for the 256-QAM example with $n_{\mathrm{ch}}=3$ and $n_{\mathrm{eq}}=15$.

that the MSE of the CMA converged approximately after 10,000 samples. The results of Fig. 10 confirm that the FL(10,000)CMA had the same steady-state performance as the CMA, but the former achieved considerably faster convergence. The SER performance of the $\mathrm{FL}(10,000)$-CMA, not shown, is similar to that of the CMA depicted in Fig. 8. The learning curve of the combined $\mathrm{FL}(10,000)-\mathrm{CMA}$ and SDD scheme, labelled as the $\mathrm{FL}(10,000)-\mathrm{CMA}+\mathrm{SDD}$, is compared with that of the CMA + SDD in Fig. 11, where it can be seen that both the blind equalisers achieved the same steady-state performance but the $\mathrm{FL}(10,000)-C M A+S D D$ converged faster than the latter, requiring approximately 4000 fewer samples to converge. The SER of the FL(10,000)-CMA + SDD, not shown, is the same as the CMA + SDD shown in Fig. 8.

\subsection{6-QAM example}

The channel length was $n_{\mathrm{ch}}=3$ with the $\mathrm{CIR} \mathbf{c}_{\mathrm{CIR}}$ given by

$$
\left[\begin{array}{lll}
-0.5+j 0.4 & 0.7-j 0.6 & 0.2+j 0.1
\end{array}\right]^{T} .
$$

The modulation scheme was 256-QAM, and the equaliser length was set to $n_{\mathrm{eq}}=15$. With the middle tap of $\mathbf{w}(0)$ initialised to $1+j 0$ and rest of the taps in $\mathbf{w}(0)$ to $0+j 0$, the blind equaliser was found to have a decision delay of $\tau=8$. Given SNR $=$ $40 \mathrm{~dB}$, the output constellation of the MMSE equaliser with $\tau=8$ is depicted in Fig. 12(a), while the output constellations of the CMA and CMA + SDD blind equalisers after convergence are illustrated in Figs. 12(b) and 12(c), respectively, where the phase rotation of the CMA $\left(-29^{\circ}\right)$ had been compensated. The step size of the CMA was set to $\mu_{\mathrm{CMA}}=2 \times 10^{-8}$ by experiment, while the appropriate step size and cluster width of the SDD were found empirically to be $\mu_{\mathrm{SDD}}=6 \times 10^{-5}$ and 


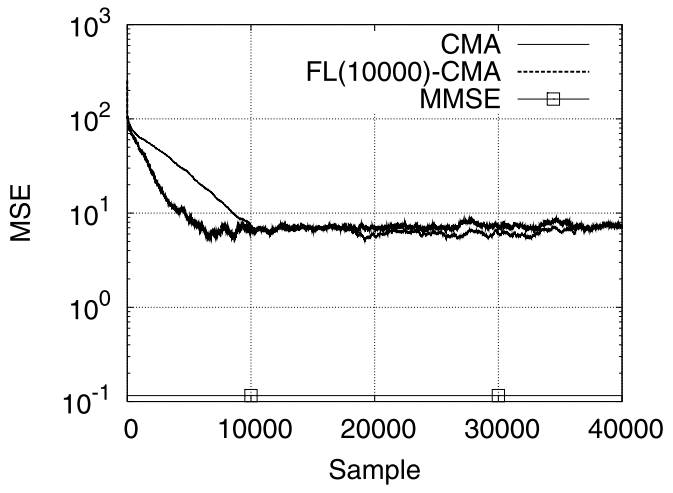

(a)

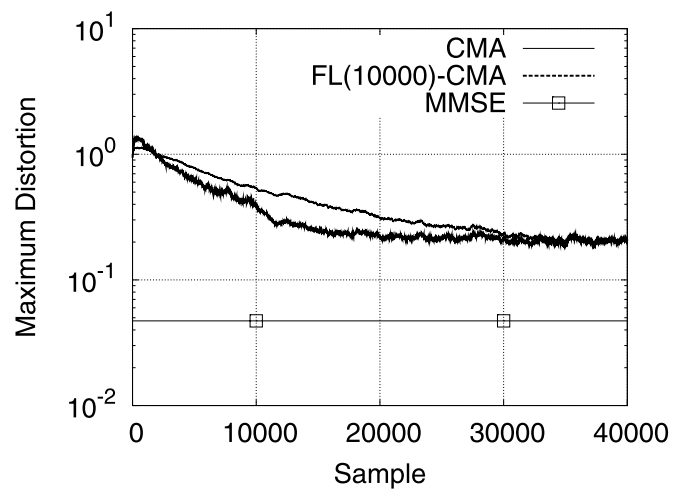

(b)

Fig. 15. Convergence performance comparison of the CMA and FL(10,000)-CMA, in terms of: (a) the MSE and (b) MD measure, averaged over 10 runs, for the 256-QAM example with $n_{\mathrm{ch}}=3, n_{\mathrm{eq}}=15$ and $\mathrm{SNR}=40 \mathrm{~dB}$. The $\mathrm{FL}(10,000)$-CMA uses the fuzzy step size for the first 10,000 samples and then switches to a constant step size.

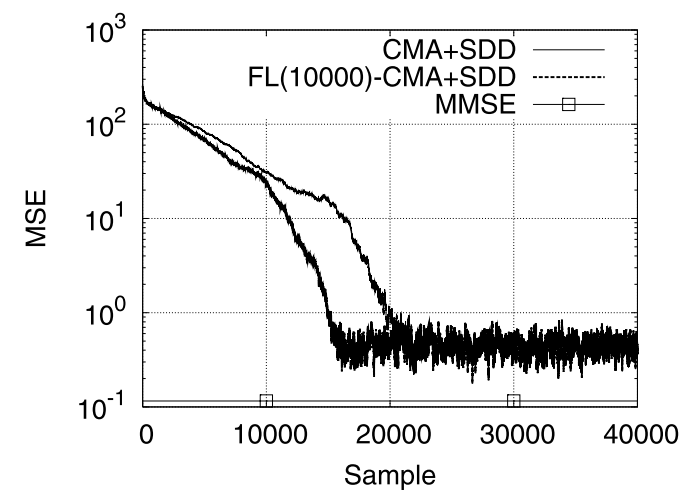

(a)

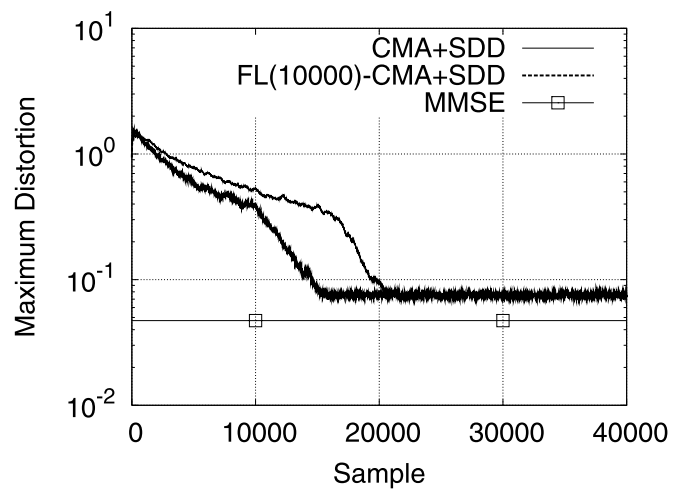

(b)

Fig. 16. Convergence performance comparison of the CMA + SDD and FL(10,000)-CMA + SDD, in terms of: (a) the MSE and (b) MD measure, averaged over 10 runs, for the 256-QAM example with $n_{\mathrm{ch}}=3, n_{\mathrm{eq}}=15$ and SNR $=40 \mathrm{~dB}$. The FL(10,000)-CMA uses the fuzzy step size for the first 10,000 samples and then switches to a constant step size.

$\rho=0.4$. The learning curves of the CMA and CMA + SDD equalisers, averaged over 10 runs, are depicted in Fig. 13, where the MSE of the CMA was calculated with the $-29^{\circ}$ phase compensation while no phase compensation was required for the CMA + SDD. The SER performance of the three equalisers, the MMSE, the CMA with the $-29^{\circ}$ phase compensation and the CMA + SDD, are compared in Fig. 14. The results of Figs. 12-14 again confirm that the CMA + SDD equaliser achieved significantly better performance than the CMA one, and its performance was close to that of the MMSE solution.

The design of the FL-CMA was summarised as follows. Since for 256 -QAM $\max |\varepsilon(k)|^{2} \approx 2 \times 10^{7}$, the centroid of $L_{\varepsilon}$ was set to $a=2 \times 10^{7}$ while the centroid of $\mathrm{P}_{\delta}$ was chosen to be $b=0.001 a=2 \times 10^{4}$. Again, the two variances of the Gaussian MBFs were chosen to be $\rho_{\varepsilon}=(0.01 a)^{2}$ and $\rho_{\delta}=(0.2 b)^{2}$, respectively. The short-term average length for calculating $\left|\varepsilon_{n}\right|^{2}$ was again set to $N_{\mathrm{sm}}=20$, while the centroid of $\mathrm{S}_{\mu}$ was chosen to be $\mu_{\min }=2 \times 10^{-8}$. This FL-CMA was used in the initial 10,000 samples of adaptation, and afterward blind adaptation was switched to the CMA with the constant step size $\mu_{\mathrm{CMA}}=2 \times 10^{-8}$. Fig. 15 compares the learning curve of the resulting $\mathrm{FL}(10,000)$-CMA with that of the CMA, while Fig. 16 depicts the learning curve of the FL(10,000)-CMA + SDD equaliser, in comparison with the CMA + SDD equaliser. The simulation results obtained again demonstrate that the $\mathrm{FL}(10,000)-\mathrm{CMA}+\mathrm{SDD}$ converged considerably faster than the CMA + SDD, requiring approximately 5000 fewer samples to converge, while both the blind equalisers had the same steady-state equalisation performance. Again, the SER of the FL(10,000)-CMA + SDD, not shown, is identical to that of the CMA + SDD plotted in Fig. 14.

\section{Conclusions}

Blind equalisation of high-order QAM systems has been revisited using the concurrent CMA and SDD scheme. The concurrent CMA and SDD scheme has been confirmed to significantly outperform the CMA, and its equalisation performance has been shown to be close to that of the MMSE equaliser with the perfect channel information. A detailed design of a fuzzy step-size CMA has been given and the advantages of using this fuzzy step size approach have been investigated. It has been 
demonstrated that, in order to achieve the maximum benefit in convergence speed and yet not to sacrifice any steady-state equalisation performance, a good strategy is to use the fuzzy step-size CMA in the initial stage of blind adaptation and to switch to the CMA with a small constant step size afterward. This switched fuzzy step-size CMA has been combined with the SDD adaptation, and the resulting concurrent blind equaliser has been shown to achieve significantly faster convergence with the same excellent steady-state equalisation performance, in comparison with the previous concurrent CMA and SDD scheme that employs a constant step size for the CMA. More specifically, the FL assisted CMA and SDD scheme requires several thousands fewer samples than the constant step-size CMA and SDD scheme to converge.

\section{References}

[1] L. Hanzo, S.X. Ng, T. Keller, W. Webb, Quadrature Amplitude Modulation: From Basics to Adaptive Trellis-Coded, Turbo-Equalised and Space-Time Coded OFDM, CDMA and MC-CDMA Systems, Wiley/IEEE Press, Chichester, UK, 2004.

[2] D. Godard, Self-recovering equalization and carrier tracking in two-dimensional data communication systems, IEEE Trans. Commun. COM-28 (1980) $1867-1875$.

[3] J.R. Treichler, B.G. Agee, A new approach to multipath correction of constant modulus signals, IEEE Trans. Acoustics Speech Signal Process. ASSP-31 (2) (1983) 459-472.

[4] J.R. Treichler, Application of blind equalization techniques to voiceband and RF modems, in: Preprints 4th IFAC Int. Symposium Adaptive Systems in Control and Signal Processing, France, 1992, pp. 705-713.

[5] N.K. Jablon, Joint blind equalization, carrier recovery, and timing recovery for high-order QAM signal constellations, IEEE Trans. Signal Process. 40 (6) (1992) 1383-1398.

[6] R. Johnson Jr., P. Schniter, T.J. Endres, J.D. Behm, D.R. Brown, R.A. Casas, Blind equalization using the constant modulus criterion: A review, Proc. IEEE 86 (10) (1998) 1927-1950.

[7] O. Macchi, E. Eweda, Convergence analysis of self-adaptive equalizers, IEEE Trans. Inf. Theor. IT-3 (2) (1984) 161-176.

[8] F.C.C. De Castro, M.C.F. De Castro, D.S. Arantes, Concurrent blind deconvolution for channel equalization, in: Proc. ICC'2001, vol. 2, Helsinki, Finland, June 11-15, 2001, pp. 366-371.

[9] A. Benveniste, M. Goursat, Blind equalizers, IEEE Trans. Commun. COM-32 (8) (1984) 871-883.

[10] S. Chen, Low complexity concurrent constant modulus algorithm and soft decision directed scheme for blind equalisation, IEE Proc. Vision Image Signal Process. 150 (5) (2003) 312-320.

[11] S. Chen, E.S. Chng, Concurrent constant modulus algorithm and soft decision directed scheme for fractionally-spaced blind equalization, in: Proc. ICC 2004, vol. 4, Paris, France, June 20-24, 2004, pp. 2342-2346.

[12] S. Chen, A. Wolfgang, L. Hanzo, Constant modulus algorithm aided soft decision directed scheme for blind space-time equalisation of SIMO channels, Signal Process. 87 (11) (2007) 2587-2599.

[13] S. Chen, S. McLaughlin, P.M. Grant, B. Mulgrew, Multi-stage blind clustering equaliser, IEEE Trans. Commun. 43 (3) (1995) $701-705$.

[14] S. Haykin, Adaptive Filter Theory, third ed., Prentice-Hall, Upper Saddle River, NJ, 1996.

[15] W.-S. Gan, Designing a fuzzy step size LMS algorithm, IEE Proc. Vision Image Signal Process. 144 (5) (1997) 261-266.

[16] J. Sanubari, Fast convergence LMS adaptive filters employing fuzzy partial updates, in: Proc. TENCON 2003, vol. 4, Oct. 15-17, 2003, pp. 1334-1337.

[17] H.-Y. Lin, C.-C. Hu, Y.-F. Chen, J.-H. Wen, An adaptive robust LMS employing fuzzy step size and partial update, IEEE Signal Process. Lett. 12 (8) (2005) $545-548$

[18] A. Özen, I. Kaya, B. Soysal, Design of a fuzzy based outer loop controller for improving the training performance of LMS algorithm, in: Proc. ICIC 2007, Qingdao, China, Aug. 21-24, 2007, pp. 1051-1063.

[19] C.-H. Cheng, J.-H. Wen, Y.-F. Chen, J.-Y. Lin, A robust interference cancellation technique for DS-UWB systems using fuzzy step size LMS algorithm, Eur. Trans. Telecommun. 19 (2) (2008) 207-217.

[20] V. Nigam, R. Priemer, Fuzzy logic based variable step size algorithm for blind delayed source separation, Fuzzy Sets Syst. 157 (13) (2006) $1851-1863$.

[21] Z. Du, S. Zhou, P. Wan, W. Wu, Novel variable step size constant modulus algorithms for blind multiuser detection, in: Proc. VTC2001 Fall, vol. 2, Oct. 7-11, 2001, pp. 673-677.

[22] J.-S. Lim, K.-Y. Han, J. Jeon, Adaptive step-size widely linear linearly constrained constant modulus algorithm for DS-CDMA receivers in nonstationary interference environments, Signal Process. 87 (6) (2007) 1523-1527.

[23] J.G. Proakis, Digital Communications, third ed., McGraw-Hill, New York, 1995.

[24] L.A. Zadeh, Fuzzy sets, Inf. Control 8 (3) (1965) 338-353.

[25] E. Cox, The Fuzzy Systems Handbook, Academic Press, London, 1994

Sheng Chen received his BEng degree from Huadong Petroleum Institute, Dongying, China, in January 1982, and PhD degree from the City University, London, UK, in September 1986, both in control engineering. He was awarded the Doctor of Sciences (DSc) degree by the University of Southampton, Southampton, UK, in 2005.

From October 1986 to August 1999, he held research and academic appointments at the University of Sheffield, the University of Edinburgh and the University of Portsmouth, all in UK. Since September 1999, he has been with the School of Electronics and Computer Science, University of Southampton, Southampton, UK. Professor Chen's research interests include wireless communications, adaptive signal processing for communications, machine learning and neural networks, finite-precision digital controller design, networked control systems, and evolutionary computation methods. He has published over 400 research papers.

Dr Chen is a Fellow of IET and a Fellow of IEEE. In the database of the world's most highly cited researchers, compiled by Institute for Scientific Information (ISI) of the USA, Dr Chen is on the list of the highly cited researchers in the engineering category.

Bing L. Luk received his BSc degree in Electrical and Electronic Engineering from the Portsmouth Polytechnic, UK, in 1985, MSc degree in Digital Computer Systems from the Brunel University, UK, in 1986 and PhD degree in Robotics from the University of Portsmouth, UK, in 1991.

From 1991 to 1999, Dr Luk was a senior lecturer with the Department of Electrical and Electronic Engineering, the University of Portsmouth. Since 2000, he has been with the Department of Manufacturing Engineering and Engineering Management at City University of Hong Kong, Hong Kong, China. Dr Luk's research interests include mobile robotics, telemedicine research, non-destructive test methods, machine learning and evolutionary computation. 
Chris J. Harris received his PhD degree from the University of Southampton, Southampton, UK, in 1972. He was awarded the Doctor of Sciences (DSc) degree by the University of Southampton in 2001.

He previously held appointments at the University of Hull, the UMIST, the University of Oxford, and the University of Cranfield, all in UK, as well as being employed by the UK Ministry of Defence. He returned to the University of Southampton as the Lucas Professor of Aerospace Systems Engineering in 1987 to establish the Advanced Systems Research Group and, more recently, Image, Speech and Intelligent Systems Group. His research interests lie in the general area of intelligent and adaptive systems theory and its application to intelligent autonomous systems such as autonomous vehicles, management infrastructures such as command \& control, intelligent control, and estimation of dynamic processes, multi-sensor data fusion, and systems integration. He has authored and co-authored 12 research books and over 400 research papers, and he is the associate editor of numerous international journals.

Dr Harris was elected to the Royal Academy of Engineering in 1996, was awarded the IEE Senior Achievement medal in 1998 for his work in autonomous systems, and the highest international award in IEE, the IEE Faraday medal, in 2001 for his work in intelligent control and neurofuzzy systems.

Lajos Hanzo received his Master degree in electronics in 1976 and his doctorate in 1983. In 2004 he was awarded the Doctor of Sciences (DSc) degree by the University Southampton, Southampton, UK.

During his 30-year career in telecommunications he has held various research and academic posts in Hungary, Germany and the UK. Since 1986 he has been with the School of Electronics and Computer Science, the University of Southampton, UK, where he holds the chair in telecommunications.

He has co-authored 20 John Wiley/IEEE Press books totalling about 20000 pages on mobile radio communications, published in excess of 800 research papers, organised and chaired conference sessions, presented overview lectures and has been awarded a number of distinctions. He is an enthusiastic supporter of industrial-academic liaison. He also offers a range of industrial research overview courses.

Professor Hanzo is a Fellow of the Royal Academy of Engineering (FREng), UK. He is an IEEE Distinguished Lecturer of both the Communications Society and the Vehicular Technology Society as well as a Fellow of both the IEEE and IEE. 